\title{
Efficacy of combinations of colistin with other antimicrobials involves membrane fluidity and efflux machinery
}

This article was published in the following Dove Press journal: Infection and Drug Resistance

\author{
E Armengol' \\ O Domenech ${ }^{2}$ \\ E Fusté ${ }^{1,3}$ \\ | Pérez-Guillén' \\ $\mathrm{JH}$ Borrell ${ }^{2}$ \\ JM Sierra' \\ M Vinas
}

'Laboratory of Molecular Microbiology and Antimicrobials, Department of Pathology and Experimental

Therapeutics, School of Medicine, University of Barcelona, Barcelona, Spain; 2Department of Pharmacy,

Pharmaceutical Technology and PhysicalChemistry, University of Barcelona, Barcelona, Spain; ${ }^{3}$ Department of Public Health, Mental Health and Perinatal Nursing. School of Nursing, University of Barcelona, Barcelona, Spain
Correspondence: JM Sierra Laboratory of Molecular Microbiology and Antimicrobials, Department of Pathology and Experimental Therapeutics, School of Medicine, IDIBELL, University of Barcelona, Feixa Llarga s/n, 08907

l'Hospitalet de Llobregat, Barcelona, Spain $\mathrm{Tel}+34934024265$

Email jmsierra@ub.edu
Objective: Despite its use was abandoned several decades ago, the polycationic peptide colistin has become the last hope to treat severe infections caused by multidrug-resistant Gram-negative bacteria. Thus, the development of colistin resistance may seriously compromise the efficacy of treatment. Moreover, colistin has high toxicity being dose dependent. A potentially effective strategy to avoid resistance may be to combine colistin with other antimicrobials. This may help in the rescue of old antimicrobials and in reducing toxic undesired effects.

Methods: Antimicrobial susceptibility determination, efflux machinery function measurements in different conditions and measurement of inhibition of the extrusion by colistin were performed. Moreover, modifications of anisotropy of the membranes by using fluorescent dyes was accomplished.

Results: Sub-inhibitory concentrations of colistin have a synergistic effect with several antimicrobials that act intracellularly (targeting protein synthesis and DNA replication). This effect was demonstrated through the uptake increases of acridine orange. in Pseudomonas aeruginosa, Escherichia coli and Acinetobacter baumanii but also in an intrinsically colistin-resistant species as Serratia marcescens. Measurements of the anisotropy of bacterial membranes, as a measure of membrane fluidity, showed significant changes indicative of colistin activity.

Conclusion: The alterations in the cellular efflux machinery that resulted in higher intracellular concentrations of acridine orange, and likely of other antimicrobials combined with data of membrane fluidity and measured synergism in vitro allow us to envisage the use of these combinations to fight infections caused by multidrug-resistant bacteria.

Keywords: synergism, cationic antimicrobial peptides, efflux pumps, membrane fluidity

\section{Introduction}

In February 2017, the World Health Organization (WHO) published a list of "critical" resistant bacterial species, including carbapenem-resistant Pseudomonas aeruginosa, Acinetobacter baumannii, and Escherichia coli. The same document urged the development of new, alternative treatments and/or new antimicrobials to reduce the number of deaths caused by multi-drug-resistant (MDR) bacteria. ${ }^{1}$

The antimicrobial peptide colistin was introduced in 1949 but in the 1980s it was withdrawn because of its nephrotoxicity and neurotoxicity. However, in the 2000s, clinicians again turned to colistin in an effort to treat infections caused by MDR Gramnegative bacteria. ${ }^{2}$ Colistin is polycationic and interacts with negatively charged membrane components, including lipopolysaccharide (LPS), to disrupt cell membranes. Other 
activities contributing to colistin's bactericidal effect are ribosome binding, alterations of bacterial respiration, disturbances of the bacterial division, the induction of structural injuries, and the production of reactive oxygen species. ${ }^{3,4}$ Nevertheless, the precise mechanisms of action of colistin and other polymyxins are not yet fully understood.

Because the toxicity of colistin is dose-dependent, ${ }^{5}$ there is considerable interest in strategies that allow dose reductions while maintaining the therapeutic efficacy of the drug. ${ }^{6}$ An additional consideration is the increasing frequency of colistin-resistant isolates. Among the resistance mechanisms identified thus far are modifications of LPS (PhoPQ and PmrAB, a two-component regulatory system) and a transferable mechanism of resistance ( $\mathrm{mcr}$ $1)^{7}$ The heterogeneous resistance (heteroresistance) of bacterial populations to colistin has also been described. However, a previous study suggested that the negative impact of colistin heteroresistance can be minimized by co-treatment with other antimicrobial agents. ${ }^{8}$

Synergism between colistin and other peptide molecules with a wide variety of antimicrobial activities has been explored. While colistin-carbapenem ( $\beta$-lactams) is probably the best-studied combination, ${ }^{9-11}$ synergies between colistin and quinolones, fosfomycin, and aminoglycosides have been reported as well. ${ }^{12}$ Moreover, even though Gram-negatives are intrinsically resistant to linezolid and glycopeptides, their use in combination with colistin may be therapeutically promising. In fact, in vitro synergisms between colistin and linezolid against A. baumannii clinical strains ${ }^{13}$ and between colistin and glycopeptides in a Galleria mellonella model of infection ${ }^{14}$ were recently demonstrated. By disrupting the outer membrane to alter cytoplasmic membrane permeability, colistin allows both linezolid and glycopeptides to penetrate Gram-negative bacteria, which are otherwise impervious to these agents. A disturbance of the bacterial membrane may also underlie the observed synergies between colistin or colistin-like peptides and other, chemically unrelated antimicrobial agents. ${ }^{15}$ Thus, in this study we examined the role of colistin synergism with other antimicrobials with respect to: i) a reduction of the colistin dose and its impact on the drug's toxicity and ii) strategies allowing the avoidance of resistance development. Since the chloramphenicol resistance of MDR bacteria is mediated by the cellular efflux machinery, the focus of our study was the effect of colistin on cellular efflux pumps. Synergy between colistin and chloramphenicol was reported by Abdul Rahim et al. ${ }^{16}$ Our results provide evidence of critical alterations in the efflux machinery of cells exposed to low concentrations of colistin and of the efficacy of other antimicrobials when administered in combination with colistin.

\section{Materials and methods}

\section{Bacterial strains, culture conditions, and media}

The 24 clinical isolates used in this study differed in their clinical origins and included 7 strains of MDR Pseudomonas aeruginosa, 9 strains of MDR Escherichia coli, and 7 of MDR Acinetobacter baumannii. None of the strains were epidemiologically related. Pseudomonas aeruginosa ATCC 27853 and PAO1, Escherichia coli ATCC 25922, and Acinetobacter baumannii ATCC 17978 served as control strains. In addition, four strains of Serratia marcescens (two environmental and two clinical isolates) were used in the accumulation experiments.

The tryptone soy agar (TSA), tryptone soy broth (TSB), and Mueller-Hinton broth (MHB) used to culture the strains were purchased from Sharlau (Sentmenat, Barcelona, Spain).

\section{Chemicals}

Colistin was kindly supplied by Zhejiang Shenghua Biok Biology Co., Ltd., (Shanghai, China), Chloramphenicol, amikacin, tetracycline, linezolid, acridine orange, the efflux pump inhibitor phenyl-arginyl- $\beta$-naphthylamide $(\mathrm{Pa} \beta \mathrm{N})$, used in the accumulation assays, and the fluorescent probes diphenylhexatriene (DPH) and 1-[4-trimethylamino-phenyl] -6-phenyl-1,3,5-hexatriene (TMA-DPH) were purchased from Sigma-Aldrich Chemicals (Madrid, Spain). The LIVE/DEAD $^{\circledR}$ BacLight $^{\mathrm{TM}}$ bacterial viability kit was from Life Technologies (Oregon, USA).

\section{Intracellular accumulation of acridine orange}

Acridine orange (AO: N,N,N',N'-tetramethylacridine -3,6-diamine) produces fluorescence after it penetrates the bacterium and binds to its DNA. It is pumped out from bacterial cells by at least one efflux pump, as shown in $E$. coll ${ }^{17}$ and other Gram-negative bacteria. ${ }^{18}$ In this study, AO uptake was determined: i) under standard culture conditions, ii) in the presence of the efflux pump inhibitor $\mathrm{Pa} \mathrm{N}^{19}(20 \mu \mathrm{g} / \mathrm{ml})$, and iii) in the presence of sub-inhibitory concentrations of colistin $(0.25$ and $0.125 \mu \mathrm{g} / \mathrm{ml})$. 
The assays were performed in flat-bottomed microtiter plates, with the same plate used for the three different conditions. Each well contained $100 \mu 1$ of the bacterial inoculun and $100 \mu \mathrm{l}$ of medium. Overnight cultures were diluted in Ringer's solution to an optical density (OD) of 1.5 at $520 \mathrm{~nm}$. The inoculated plates were incubated for $1 \mathrm{~h}$, after which fluorescence was measured in a FLUOstar OPTIMA Biogen fluorescent microplate reader. The results were expressed as the percentage increase in fluorescence vs that in wells containing bacteria and $\mathrm{AO}(=100 \%)$.

\section{Susceptibility test}

The microdilution method was used to determine the minimum inhibitory concentrations (MICs) of colistin, chloramphenicol, tetracycline, vancomycin, and linezolid in each of the strains, according to EUCAST recommendations. Susceptibility was defined following EUCAST definition of antimicrobial breakpoints. ${ }^{20}$ The MIC was also determined in the presence of the efflux pump inhibitor $\mathrm{Pa} \beta \mathrm{N}$. Bacterial susceptibility to several different antimicrobials (chloramphenicol, tetracycline, vancomycin, and linezolid) in the presence of sub-inhibitory concentrations of colistin was evaluated as well.

\section{Fluorescent live/dead testing}

To rule out bacterial death during the accumulation experiment and to show that $\mathrm{AO}$ fluorescence was due solely to an increase in the uptake of the dye, the viability of the bacteria in the presence of different concentrations of colistin was determined in a live-dead analysis.

Briefly, $1 \mathrm{ml}$ of bacterial suspension (prepared as described above for the AO accumulation experiment) was incubated with colistin and centrifuged for $5 \mathrm{~min}$ at $18,000 \times \mathrm{g}$ and then stained with $100 \mu \mathrm{l}$ of the Live/Dead kit solution (LIVE/DEAD ${ }^{\circledR}$ BacLight $^{\mathrm{TM}}$ ). The cells were subsequently examined by confocal laser scanning microscopy (CLSM) using a ZEISS LSM 880 microscope equipped with a $488 \mathrm{~nm}$ argon laser, $561 \mathrm{~nm}$ and $633 \mathrm{~nm}$ diode lasers, and a $63 \times$ magnification oil immersion objective. All experiments were performed in duplicate. CLSM images were analyzed using the ZEN 2.3 software. The proportions of living vs dead bacteria were calculated.

\section{Membrane polarization and anisotropy}

Bacterial cultures with or without colistin were washed twice in buffer and then suspended in saline buffer to a final $\mathrm{OD}_{500}$ of 0.4. After the addition of DPH or TMA-DPH to a final concentration of $2 \mu \mathrm{M}$ and $1 \mu \mathrm{M}$, respectively, the cultures were incubated for $15 \mathrm{~min}$ at $37{ }^{\circ} \mathrm{C}$ to enhance incorporation of the probes into the bacterial membranes. Anisotropy measurements were performed in cells incubated for $1 \mathrm{~h}$ in the presence of colistin.

Fluorescence was measured using a SLM-Aminco 8100 spectrofluorometer with a thermal jacketed cuvette holder. Fluorescence anisotropy $(r)$ was calculated as:

$$
r=\frac{I_{V V}-G \cdot I_{V H}}{I_{V V}+2 G \cdot I_{V H}} ; G=\frac{I_{V H}}{I_{H H}}
$$

where $I_{\mathrm{ij}}$ is the fluorescence intensity measured when the excitation $(i)$ and emission $(j)$ polarizers are fixed in the vertical $(V)$ or horizontal $(H)$ position and $G$ is the ratio of the sensitivities of the detection system for vertically and horizontally polarized light. Samples fluorescence emission was measured at $37{ }^{\circ} \mathrm{C}$ using excitation and emission wavelengths of $381 \mathrm{~nm}$ and $426 \mathrm{~nm}$, respectively. ${ }^{21}$

\section{Results}

\section{Susceptibility testing}

The MICs for the antimicrobials investigated in this study are shown in Table 1. All tested strains were susceptible to colistin according to the breakpoint defined by EUCAST. Table 1 also shows the effect of sub-inhibitory concentrations of colistin on the MICs of other antimicrobials. The activities of all antimicrobials tested increased when used in combination with colistin at $1 / 2$ of its MIC. The greatest reduction in the MICs of these drugs was obtained in A. baumannii, with moderate reductions determined in $P$. aeruginosa and $E$. coli. No significant deviations were measured in the different series of experiments.

\section{Acridine orange accumulation}

Acridine orange penetrates Gram-negative bacteria easily, although it is normally expelled by efflux pumps located in the bacterial membrane. ${ }^{22}$ However, as shown in Figure $1 \mathrm{~A}-\mathrm{C}$, in the presence of $\mathrm{Pa} \beta \mathrm{N}$, an efflux pump inhibitor, the uptake of AO increased by nearly $200 \%$ in E. coli and by $\sim 170 \%$ in $P$. aeruginosa and A. baumannii. Similar effects were achieved with sub-inhibitory concentrations of colistin $(0.25 \mu \mathrm{g} / \mathrm{ml})$, which increased $\mathrm{AO}$ uptake in $E$. coli by $\sim 160 \%$, in $P$. aeruginosa by $140 \%$, and in $A$. baumannii by $>125 \%$. The accumulation of AO was concentration-dependent, at least at the colistin concentrations tested $(0.125$ and $0.25 \mu \mathrm{g} / \mathrm{ml})$. Moreover, low concentrations of colistin also increased AO accumulation by Serratia marcescens, a bacterium intrinsically resistant to colistin (Figure 1D). Accumulation values 
Table I Bacterial susceptibility to antimicrobials administered either alone or in combination with colistin at $1 / 2$ its MIC

\begin{tabular}{|c|c|c|c|c|c|c|c|c|c|}
\hline Acinetobacter baumannii & COL & CHL & $\mathrm{CHL}+\mathrm{COL}$ & TET & TET+COL & LZD & LZD+COL & VAN & VAN+COL \\
\hline ABAU 137 & I & 64 & $\leq 0.125$ & $>128$ & I & $>256$ & 4 & $>256$ & I \\
\hline ABAU 80 & I & 64 & 0.5 & $>128$ & I & $>256$ & 8 & 256 & 2 \\
\hline ABAU 226 & I & 128 & 0.5 & 4 & I & $>256$ & 16 & $>256$ & 8 \\
\hline ATCC 17978 & I & 64 & 0.5 & 2 & 0.25 & $>256$ & 16 & 256 & 4 \\
\hline ABAU 8 & I & 64 & $\leq 0.125$ & $>128$ & 0.5 & $>256$ & 16 & 256 & 1 \\
\hline ABAU RUH-875 & I & $>128$ & 0.25 & 32 & 0.5 & $>256$ & 8 & 128 & 4 \\
\hline ABAU 176 & I & 128 & 0.5 & $>128$ & I & $>256$ & 16 & 256 & 8 \\
\hline ABAU 34 & 0.5 & 64 & 0.5 & $>128$ & 2 & $>256$ & 16 & $>256$ & 2 \\
\hline \multicolumn{10}{|l|}{ Pseudomonas aeruginosa } \\
\hline PA 362 & 0.25 & 64 & 4 & 4 & 2 & $>256$ & 64 & 256 & 2 \\
\hline PA 350 & 0.5 & 8 & 4 & 8 & 4 & 256 & 32 & 128 & 2 \\
\hline PA 056 & 0.5 & 64 & 2 & 16 & 4 & $>256$ & 32 & $>256$ & 8 \\
\hline PA 846 & 0.25 & 8 & 4 & 4 & 0.5 & 128 & 16 & 128 & 8 \\
\hline PA OI & 0.5 & 16 & 2 & 8 & 4 & $>256$ & 32 & 128 & 16 \\
\hline PA 023 & 0.5 & 8 & 0.25 & 16 & 2 & $>256$ & 64 & $>256$ & 8 \\
\hline PA 666 & I & 128 & 2 & 16 & 2 & $>256$ & 32 & 128 & 16 \\
\hline PA 086 & 0.5 & 8 & 0.5 & 8 & I & $>256$ & 64 & 256 & 16 \\
\hline ATCC 27853 & 0.5 & 64 & 4 & 128 & I & $>256$ & 64 & 256 & 8 \\
\hline \multicolumn{10}{|l|}{ Escherichia coli. } \\
\hline MDR 211453 & 0.5 & 4 & 4 & I & I & 256 & 32 & 256 & 8 \\
\hline MDR 39255 & 0.5 & 4 & I & 128 & 64 & 256 & 32 & 256 & 8 \\
\hline MDR 20869I & 0.5 & $>128$ & 2 & $>128$ & 32 & 256 & 32 & 256 & 8 \\
\hline MDR 205II9 & 0.5 & 64 & I & $>128$ & 16 & 128 & 16 & 128 & 16 \\
\hline MDR 2464 I 5 & I & $>128$ & 16 & $>128$ & 2 & 256 & 32 & 128 & 8 \\
\hline MDR 239910 & I & 16 & 2 & 128 & 16 & 256 & 16 & 256 & 8 \\
\hline MDR 238192 & 0.5 & 128 & 32 & $>128$ & 32 & 256 & 16 & 256 & 16 \\
\hline MDR 2 I5482 & I & $>128$ & 64 & $>128$ & 16 & $>256$ & 8 & $>256$ & 4 \\
\hline MDR $2 / 3873$ & I & 4 & 4 & 128 & 16 & 128 & 64 & 64 & 16 \\
\hline ATCC 25922 & 0.5 & I & I & I & 0,25 & 128 & 16 & 128 & 16 \\
\hline
\end{tabular}

Note: MICs are reported in $\mu \mathrm{g} / \mathrm{ml}$.

Abbreviations: COL, colistin; CHL, chloramphenicol; TET, tetracycline; LZD, linezolid; VAN, vancomycin.

obtained were highly repetitive. In Figure 1 standard deviations are represented.

To rule out that these results reflected differences in bacterial viability, a live/dead assay was conducted and the results were evaluated by CLSM (Figure 2). Bacterial death in the presence of colistin was $\sim 6 \%$, the same as in the control (without colistin). Thus, at the tested subinhibitory concentrations, the membrane effects of colistin did not cause a reduction in cell viability. In addition, propidium iodide was unable to penetrate the colistintreated bacteria.

\section{Membrane polarization}

Anisotropy is the inverse of microviscosity. It can be measured in membranes using the hydrophobic probe $\mathrm{DPH}$, which enters the inner domain of the lipid bilayer and becomes lodged parallel to the hydrocarbon chain axis of the phospholipids but also in the centre of the bilayer, parallel to the surface. Trimethylammonium (TMA)-DPH, the cationic derivative of DPH, localizes closer to the external surface of the lipid membrane, as the cationic TMA substituent acts as a surface anchor. Table 2 shows the DPH- and TMA-DPH-based anisotropy values in the three tested species of bacteria incubated with 2 and $0.25 \mu \mathrm{g}$ colistin $/ \mathrm{ml}$. In E. coli MDR208691, a $1 \mathrm{~h}$ incubation with colistin reduced membrane anisotropy, while in P.aeruginosa and A. baumannii the reductions were lower (see DPH results in Table 2). Using the TMA_DPH probe, subtle effects of colistin on the membrane of $E$. coli and $P$. aeruginosa were seen, while in A. baumannii colistin slightly rigidified the surface of the lipid membrane. 
A

Escherichia coli

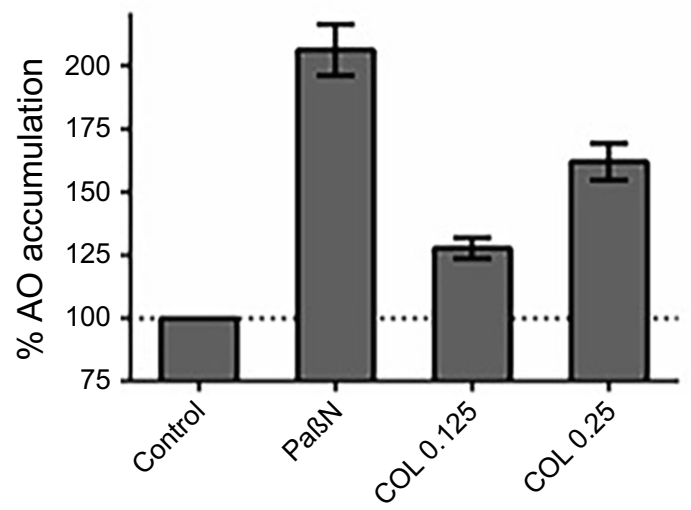

C

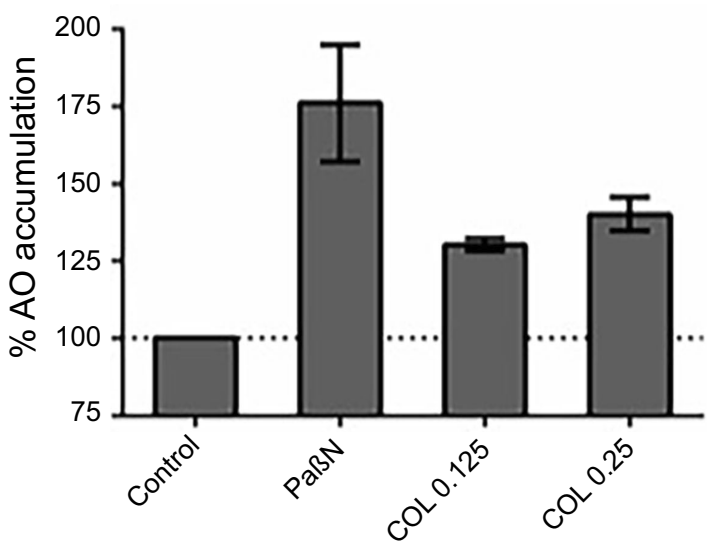

B Acinetobacter baumannii

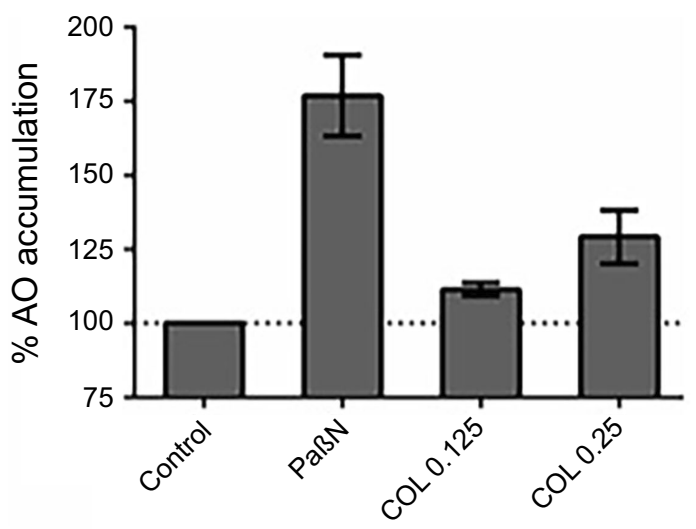

D Serratia marcescens

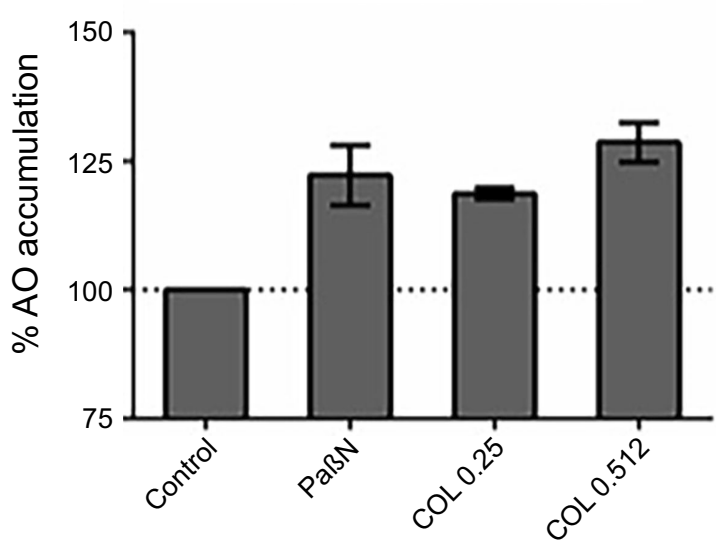

Figure I Average acridine orange (AO) accumulation in the presence of the efflux inhibitor $\mathrm{Pa} \beta \mathrm{N}$ and sub-inhibitory concentrations of colistin. (A-C) colistin-sensitive strains; (D) a species intrinsically resistant to colistin.

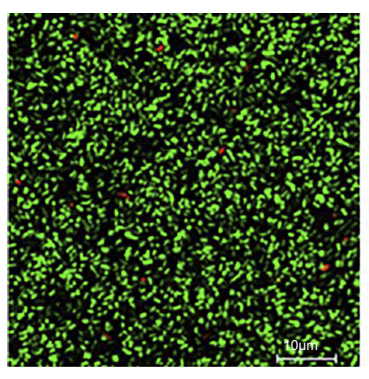

A

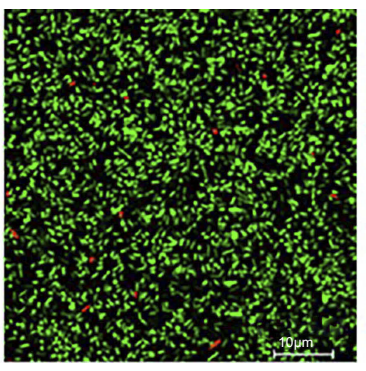

B

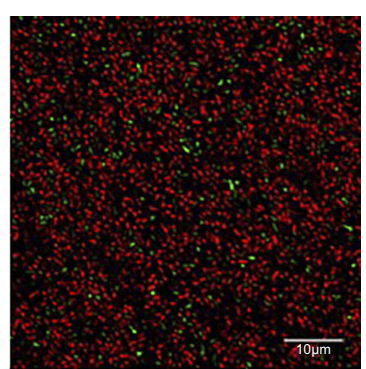

C

Figure 2 Confocal light microscopy imaging after Live/Dead staining of Pseudomonas aeruginosa. (A) Untreated cells; (B) cells treated with colistin at $0.25 \mu \mathrm{gg} / \mathrm{mL}$ and $(\mathbf{C})$ cells treated with colistin at bactericidal concentration. Similar images were obtained for Acinetobacter baumanii and E. coli (not shown). TEM of thin sections of $S$. marcescens after treatment with colistin. Blebs formed by the outer membrane are easily visualized.

\section{Discussion}

Although the toxicity of colistin resulted in the eventual discontinuation of its use as an antibiotic, with the emergence of bacterial multi-resistance the drug has been resurrected for the treatment of infections by MDR bacteria. However, the re-introduction of colistin has been accompanied by the appearance of resistance to colistin and other polymyxins, most commonly via LPS modification. A further challenge is the heteroresistance of bacterial populations; that is, the presence of resistant subpopulations within an isolate considered susceptible. $^{6,7}$ 
Table 2 Changes in membrane fluidity (anisotropy) in the presence of colistin

\begin{tabular}{|c|c|c|c|}
\hline & \multirow[t]{2}{*}{ Colistin $(\mu \mathrm{g} / \mathrm{ml})$} & \multicolumn{2}{|l|}{ Anisotropy (r) } \\
\hline & & DPH & TMA-DPH \\
\hline \multirow[t]{3}{*}{ E. coli } & 0 & $0.2787 \pm 0.0219$ & $0.267 \pm 0.039$ \\
\hline & 0.25 & $0.26986 \pm 0.020$ & $0.265 \pm 0.028$ \\
\hline & 2.00 & $0.2488 \pm 0.009$ & $0.2681 \pm 0.0212$ \\
\hline \multirow[t]{3}{*}{ P. aeruginosa } & 0 & $0.3139 \pm 0.034$ & $0.284 \pm 0.0247$ \\
\hline & 0.25 & $0.3105 \pm 0.0359$ & $0.274 \pm 0.0199$ \\
\hline & 2.00 & $0.2877 \pm 0.0213$ & $0.273 \pm 0.0212$ \\
\hline \multirow[t]{3}{*}{ A. baumannii } & 0 & $0.314 \pm 0.027$ & $0.255 \pm 0.005$ \\
\hline & 0.25 & $0.306 \pm 0.029$ & $0.260 \pm 0.008 \mathrm{I}$ \\
\hline & 2.00 & $0.307 \pm 0.0025$ & $0.260 \pm 0.006$ \\
\hline
\end{tabular}

To avoid the development of colistin resistance, the colistin is used in combination with a second antimicrobial agent, based on the advantage conferred by the main mechanism of colistin action: membrane permeabilization. Ritcher et $\mathrm{al}^{23}$ described the increased accumulation of several antimicrobials in the presence of high concentrations of colistin ( $6 \mathrm{mM}=7 \mu \mathrm{g} / \mathrm{ml})$. In our study, sub-MIC concentrations of colistin were used to explore their ability to enhance the action of other antibiotics while minimizing the risk posed by colistin toxicity. The results showed that sub-MIC concentrations of colistin altered the efflux pump function of the bacterial cells, as evidenced by the increases in AO accumulation in isolates of E. coli, A. baumannii, and $P$. aeruginosa. All the three species are naturally susceptible to colistin, which at concentrations at or above its $\mathrm{MIC}$ causes catastrophic effects on the outer membrane. Moreover, similar results were obtained in S. marcescens, a Gram-negative species that is naturally colistin-resistant. Specifically, we were able to demonstrate severe alterations in AO efflux in $S$. marcescens cells treated with subinhibitory concentrations of colistin. This result suggests a mechanism of outer membrane injury in Gram-negative bacteria in which correct formation of the AcrAB-tolC complex and other efflux-pump-based complexes is prevented and efflux is therefore interrupted.

Diffusion and active transport by efflux systems are strongly dependent on membrane fluidity. ${ }^{24}$ Vincent et $\mathrm{al}^{25}$ described changes in membrane polarization in bacterial cells exposed to tetracycline. As the main mechanism of action of colistin is membrane disruption, we hypothesized that concentrations of the drug below the MIC would be sufficient to alter membrane fluidity. Our results showed that low concentrations of colistin significantly increased the fluidity of the inner regions of the lipid bilayers without significantly modifying the more polar regions of the membrane, as indicated by anisotropy measurements. These colistin-induced membrane alterations would presumably i) facilitate the penetration of other antimicrobials and thus increase their biological activity and ii) decrease the lateral lipid pressure inside lipid membranes to reduce local rigidity and modify the correct packing of the efflux pumps, thus altering the efflux of drugs reaching the cell, as observed in Figure 1.

Using a live/dead assay, we showed that, at $1 / 2$ its MIC, colistin did not cause bacterial death or injury to the cytoplasmic membrane, as the amount of propidium iodide and thus the proportion of living bacteria were identical in colistin-treated and untreated (control) cells. Therefore, the above-described results cannot be explained by differences in bacterial viability.

The cellular accumulation of AO increases in the presence of the efflux pump inhibitor $\mathrm{Pa} \beta \mathrm{N}$ since the dye is normally pumped out by efflux systems. Sub-inhibitory concentrations of colistin also led to increases in the amount of $\mathrm{AO}$ retained by the bacteria. Given the results of the live/ dead test, the observed accumulation of AO can be attributed to the disruption of membrane permeability by colistin and to the indirect inhibition of the cellular efflux machinery.

We then demonstrated that sub-inhibitory concentrations of colistin increase the activity of antimicrobials whose mechanisms of resistance are associated with efflux systems and/or decreased permeability (Table 1). The synergy between clinically relevant concentrations of colistin and chloramphenicol in Klebsiella pneumoniae ${ }^{16}$ as well as between colistin and colistin-like peptides and carbapenems in imipenem-resistant $P$. aeruginosa ${ }^{15}$ has 
been reported. An enhancement of the activity of glycopeptides targeting A. baumannii was also shown in vivo, in the moth Galleria mellonella, in which colistin increased the antimicrobial activity of vancomycin by up to $90 \%{ }^{14,26}$ Moreover, Liu et $\mathrm{al}^{13}$ reported the efficacy of anti-Gram-positive antimicrobials in the treatment of A. baumannii infections when these drugs were administered together with colistin. During the writing of the present article, Brennan-Krohn et $\mathrm{al}^{27}$ published a study demonstrating the synergism of colistin and a wide range of antimicrobials in both colistin-susceptible and colistinresistant Enterobacteriaceae. The authors attributed these results to the direct effect of colistin on the bacterial outer membrane. Almost simultaneously, Fang et $\mathrm{al}^{28}$ reported similar data in tests of $E$. coli exposed to combinations of colistin and either azithromycin or rifampin, both in vitro and in vivo. Our data provide additional, biophysical evidence supporting those observations. Furthermore, these results together suggest lines of further research aimed at the development of compounds that, while unable to kill bacteria themselves, significantly alter bacterial membrane fluidity and thus potentiate the activity of traditional antimicrobials.

In summary, our study showed that, by altering the permeability of the outer membrane of Gram-negative bacteria, colistin acts synergistically with a broad spectrum of antimicrobials. The mechanism of action involves modification of membrane fluidity as well as the direct partial inhibition of efflux pump function.

\section{Acknowledgments}

The authors thank Dr. Benjamin Torrejón, from the Scientific and Technological Centers of the University of Barcelona (Bellvitge Campus), for his support in CLSM imaging. This work was financed by funding from the University of Barcelona. MV is a member of the ENABLE consortium. The research of this team is supported by the TV3-Marato Foundation (2018).

\section{Author contributions}

All authors contributed to data analysis, drafting or revising the article, gave final approval of the version to be published, and agree to be accountable for all aspects of the work.

\section{Disclosure}

The authors have no conflicts of interest to declare in this work.

\section{References}

1. WHO world health organization. WHO publishes list of bacteria for which new antibiotics are urgently needed. WHO. Available from: http://www.who.int/mediacentre/news/releases/2017/bacteriaantibiotics-needed/en/. Published 2017. Accessed June 25, 2019.

2. Bialvaei AZ, Samadi Kafil H. Colistin, mechanisms and prevalence of resistance. Curr Med Res Opin. 2015;31(4):707-721. doi:10.1185/ 03007995.2015.1018989

3. Sierra JM, Fusté E, Rabanal F, Vinuesa T, Viñas M. An overview of antimicrobial peptides and the latest advances in their development. Expert Opin Biol Ther. 2017;17(6):663-676. doi:10.1080/ 14712598.2017.1315402

4. Trimble MJ, Mlynárčik P, Kolář M, Hancock REWW. Polymyxin: alternative mechanisms of action and resistance. Cold Spring Harb Perspect Med. 2016;6(10):cshperspect.a025288. doi:10.1101/cshperspect.a025288

5. Karaiskos I, Souli M, Galani I, Giamarellou H. Colistin: still a lifesaver for the 21st century? Expert Opin Drug Metab Toxicol. 2017;13(1):59-71. doi:10.1080/17425255.2017.1230200

6. Lim LM, Ly N, Anderson D, et al. Resurgence of colistin: A review of resistance, toxicity, pharmacodynamics, and dosing. Pharmacotherapy. 2010;30(12):1279-1291. doi:10.1592/phco.30. 12.1279

7. Srinivas P, Rivard K. Polymyxin resistance in Gram-negative pathogens. Curr Infect Dis Rep. 2017;19(11):38. doi:10.1007/ s11908-017-0596-3

8. Lenhard JR, Nation RL, Tsuji BT. Synergistic combinations of polymyxins. Int $J$ Antimicrob Agents. 2016;48(6):607-613. doi:10.1016/j.ijantimicag.2016.09.014

9. Yang H, Chen G, Hu L, et al. Enhanced efficacy of imipenem-colistin combination therapy against multiple-drug-resistant Enterobacter cloacae : in vitro activity and a Galleria mellonella model. $J$ Microbiol Immunol Infect. 2018;51(1):70-75. doi:10.1016/j. jmii.2016.01.003

10. D'Souza BB, Padmaraj SR, Rekha PD, Tellis RC, Prabhu S, Pothen P. In vitro synergistic activity of colistin and ceftazidime or ciprofloxacin against multidrug-resistant clinical strains of Pseudomonas aeruginosa. Microb Drug Resist. 2014;20(6):550-554. doi:10.1089/mdr.2014.0006

11. Rudilla H, Pérez-Guillén I, Rabanal F, Sierra JM, Vinuesa T, Viñas M. Novel synthetic polymyxins kill Gram-positive bacteria. $J$ Antimicrob Chemother. 2018;73(12):3385-3390. doi:10.1093/jac/dky366

12. Ni W, Shao X, Di X, Cui J, Wang R, Liu Y. In vitro synergy of polymyxins with other antibiotics for Acinetobacter baumannii: a systematic review and meta-analysis. Int $J$ Antimicrob Agents. 2015;45(1):8-18. doi:10.1016/j.ijantimicag.2014.10.002

13. Liu B, Liu Y, Di X, et al. Colistin and anti-Gram-positive bacterial agents against Acinetobacter baumannii. Rev Soc Bras Med Trop. 2014;47(4):451-456. doi:10.1590/0037-8682-0081-2014

14. Hornsey M, Wareham DW. In vivo efficacy of glycopeptide-colistin combination therapies in a Galleria mellonella model of Acinetobacter baumannii infection. Antimicrob Agents Chemother. 2011;55(7):3534-3537. doi:10.1128/AAC.00230-11

15. Rudilla H, Fusté E, Cajal Y, Rabanal F, Vinuesa T, Viñas M. Synergistic antipseudomonal effects of synthetic peptide AMP38 and carbapenems. Molecules. 2016;21(9):1223. doi:10.3390/molecules21091223

16. Abdul Rahim N, Cheah S-E, Johnson MD, et al. Synergistic killing of NDM-producing MDR Klebsiella pneumoniae by two "old" antibiotics-polymyxin B and chloramphenicol. J Antimicrob Chemother. 2015;70(9):2589-2597. doi:10.1093/jac/dkv135

17. Ma D, Cook DN, Alberti M, Pon NG, Nikaido H, Hearst JE. Genes acrA and acrB encode a stress-induced efflux system of Escherichia coli. Mol Microbiol. 1995;16(1):45-55. doi:10.1111/j.13652958.1995.tb02390.x 
18. Sánchez L, Pan W, Viñas M, Nikaido H. The acrAB homolog of Haemophilus influenzae codes for a functional multidrug efflux pump. $J$ Bacteriol. 1997;179(21):6855-6857. doi:10.1128/ jb.179.21.6855-6857.1997

19. Renau TE, Léger R, Flamme EM, et al. Inhibitors of efflux pumps in Pseudomonas aeruginosa potentiate the activity of the fluoroquinolone antibacterial levofloxacin. $J$ Med Chem. 1999;42 (24):4928-4931. doi:10.1021/jm9904598

20. EUCAST. European committee on antimicrobial susceptibility testing breakpoint tables for interpretation of MICs and zone diameters version 6.0, valid from 2016-01-01. Available from: http://www. eucast.org/fileadmin/src/media/PDFs/EUCAST_files/Breakpoint_ tables/v_9.0_Breakpoint_Tables.xlsx. Accessed January, 2019.

21. Mykytczuk NCS, Trevors JT, Leduc LG, Ferroni GD. Fluorescence polarization in studies of bacterial cytoplasmic membrane fluidity under environmental stress. Prog Biophys Mol Biol. 2007;95(1-3):60-82. doi:10.1016/j.pbiomolbio.2007.05.001

22. Martins A, Amaral L. Screening for efflux pump systems of bacteria by the new acridine orange agar method. In Vivo (Brooklyn). 2012;26 (2):203-206.

23. Richter MF, Drown BS, Riley AP, et al. Predictive compound accumulation rules yield a broad-spectrum antibiotic. Nature. 2017;545 (7654):299-304. doi:10.1038/nature22308
24. Eze MO, McElhaney RN. The effect of alterations in the fluidity and phase state of the membrane lipids on the passive permeation and facilitated diffusion of glycerol in Escherichia coli. J Gen Microbiol. 1981;124(2):299-307. doi:10.1099/00221287-124-2-299

25. Vincent M, England LS, Trevors JT. Cytoplasmic membrane polarization in Gram-positive and Gram-negative bacteria grown in the absence and presence of tetracycline. Biochim Biophys Acta - Gen Subj. 2004;1672(3):131-134. doi:10.1016/j.bbagen.2004.03.005

26. Wareham DW, Gordon NC, Hornsey M. In vitro activity of teicoplanin combined with colistin versus multidrug-resistant strains of Acinetobacter baumannii. $J$ Antimicrob Chemother. 2011;66 (5):1047-1051. doi:10.1093/jac/dkr069

27. Brennan-Krohn T, Pironti A, Kirby JE. Synergistic activity of colistin-containing combinations against colistin-resistant enterobacteriaceae. Antimicrob Agents Chemother. 2018;62(10): AAC.00873-18. doi:10.1128/AAC.00873-18

28. Li Y, Lin X, Yao X, et al. Synergistic antimicrobial activity of colistin in combination with rifampin and azithromycin against Escherichia coli producing MCR-1. Antimicrob Agents Chemother. 2018;62(12): e1631-e18. doi:10.1128/AAC.01631-18
Infection and Drug Resistance

\section{Publish your work in this journal}

Infection and Drug Resistance is an international, peer-reviewed openaccess journal that focuses on the optimal treatment of infection (bacterial, fungal and viral) and the development and institution of preventive strategies to minimize the development and spread of resistance. The journal is specifically concerned with the epidemiology of antibiotic resistance and the mechanisms of resistance development and diffusion in both hospitals and the community. The manuscript management system is completely online and includes a very quick and fair peerreview system, which is all easy to use. Visit http://www.dovepress.com/ testimonials.php to read real quotes from published authors. 